\title{
INFRARED SPECTROSCOPY OF NOVA V705 CAS
}

\author{
A. EVANS ${ }^{1}$, T. R. GEBALLE ${ }^{2}$, J. M. C. RAWLINGS ${ }^{3}$, \\ A. D. SCOTT \\ 1. Department of Physics, Keele University, UK \\ 2. Joint Astronomy Centre, Hilo, Hawaii, USA \\ 3. Department of Physics \& Astronomy, University College, \\ London, UK
}

\section{Introduction}

Nova V705 Cas was discovered on 1993 December 7, several days before maximum light. The light curve indicates that $t_{2} \simeq 40 \mathrm{~d}$ (see e.g. [4]), characteristic of dust-forming novae. The light curve went into decline on day 58 , indicating the formation of an optically thick dust shell. IR spectra have been obtained regularly since outburst using the cooled grating spectrometer CGS4 on the United Kingdom InfraRed Telescope (UKIRT). We present here the results of ongoing IR spectroscopic monitoring of this nova.

\section{Early detection of $\mathbf{C O}$}

Emission by $\mathrm{CO}$ in the $\mathrm{K}$-band was detected around maximum light (see Fig. 1a), the earliest detection of a molecule in the ejecta of an erupting nova. The presence of $\mathrm{CO}$ points to the presence of a cool, neutral region at the outer edge of the ejecta, in which chemical reactions occur. Assuming that the $\mathrm{CO}$ is optically thin, we find a $\mathrm{CO}$ mass of $2.810^{-10} \mathrm{M}_{\odot}$, temperature $4300 \mathrm{~K}$, and that $\sim 10^{-4}$ (by number) of the $\mathrm{C}$ in the ejecta was in the form of $\mathrm{CO}$ [1]. The time-scale for forming $\mathrm{CO}$ in nova ejecta, as per the chemical models of $[5,6]$, is a few hours. The presence of $\mathrm{CO}$, and its characteristics, provide strong support for these models.

\section{UIR emission}

The so-called Unidentified InfraRed (UIR) features were first seen on day 252 (see Fig. 1b); they were not present on day 158. They are different to those appearing in other Galactic UIR sources, such as the Orion Bar. 

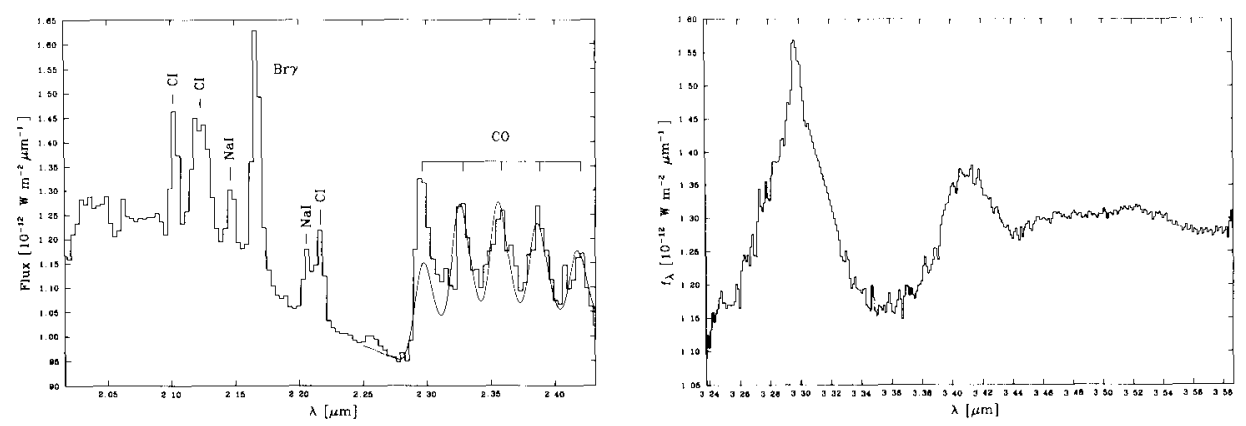

Figure 1. (a - left): CO emission at day -1 ; curve is model fit to optically thin CO emission. (b - right): UIR emission in the $3 \mu \mathrm{m}$ band at day 252 .

The $3.4 \mu \mathrm{m}$ feature is anomalously strong by comparison with the $3.28 \mu \mathrm{m}$ feature; the $11.3 \mu \mathrm{m}$ feature is also present, and is accompanied by a $8 \mu \mathrm{m}$ feature, not normally associated with UIR emission. Previous work [2] has argued that, in novae, these features arise in hydrogenated carbon grains rather than free-flying PAH molecules and that, in view of the abundance anomalies in novae, nova UIR features would differ substantially from those normally seen in Galactic objects such as the Orion Bar.

\section{Line emission}

The early spectrum is dominated by low excitation emission lines, particularly $\mathrm{CI}_{\mathrm{I}}$. Later spectra reveal the presence of [Si vI] $\lambda 1.96,2.22$ and [NeII] $\lambda 12.8$. However there is no evidence that this nova displayed a coronal phase similar to that displayed by other recent novae [3].

Spectroscopic monitoring, at near-IR $(J H K L)$ and at $10 \mu \mathrm{m}$, is continuing, and will be supplemented by ISO observations in due course.

Acknowledgements. We thank UKIRT observers for taking the early spectra of Nova Cas. UKIRT is operated by the U.K. PPARC.

\section{References}

1. Evans, A., Geballe, T.R., Rawlings, J.M.C., Scott, A. D., 1996, MNRAS, in press

2. Evans, A., Rawlings, J.M.C., 1994, MNRAS, 269, 427

3. Greenhouse, M.A., Grasdalen, G.L., Woodward, C.E. et al., 1990, Ap. J., 352, 307

4. Petrík, K., Hric, L., Urban, Z., et al., 1996, these proceedings, p323

5. Rawlings, J.M.C., 1988, MNRAS, 232, 507

6. Rawlings, J.M.C., Williams, D.A., 1989, MNRAS, 240, 729 